\title{
Structural Characterization of Native and Modified Encapsulins as Nanoplatforms for in Vitro Catalysis and Cellular Uptake
}

Rindia M. Putri, ${ }^{\dagger}$ Carolina Allende-Ballestero, ${ }^{\ddagger}$ Daniel Luque, ${ }^{\ddagger}, \S$ Robin Klem, ${ }^{\dagger}$ Katerina-Asteria Rousou, ${ }^{\dagger}$

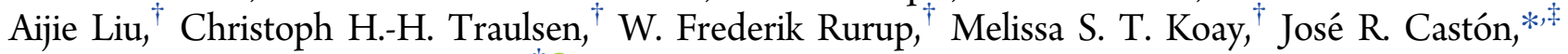
and Jeroen J. L. M. Cornelissen ${ }^{*}{ }^{\dagger}$

${ }^{\dagger}$ Department of Biomolecular Nanotechnology, MESA+ Institute for Nanotechnology, University of Twente, 7500 AE Enschede, The Netherlands

${ }^{\ddagger}$ Department of Structure of Macromolecules, Centro Nacional de Biotecnología/CSIC, Cantoblanco, 28049 Madrid, Spain

${ }^{\S}$ Centro Nacional de Microbiología/Instituto de Salud Carlos III, Majadahonda, 28220 Madrid, Spain

\section{Supporting Information}

ABSTRACT: Recent years have witnessed the emergence of bacterial semiorganelle encapsulins as promising platforms for bio-nanotechnology. To advance the development of encapsulins as nanoplatforms, a functional and structural basis of these assemblies is required. Encapsulin from Brevibacterium linens is known to be a protein-based vessel for an enzyme cargo in its cavity, which could be replaced with a foreign cargo, resulting in a modified encapsulin. Here, we characterize the native structure of $B$. linens encapsulins with both native and foreign cargo using cryo-electron microscopy (cryo-EM). Furthermore, by harnessing the confined enzyme (i.e., a peroxidase), we demonstrate the functionality of the encapsulin for an in vitro surface-immobilized catalysis in a cascade pathway with an additional enzyme, glucose oxidase. We also demonstrate the in vivo functionality of the encapsulin for cellular uptake using mammalian macrophages. Unraveling

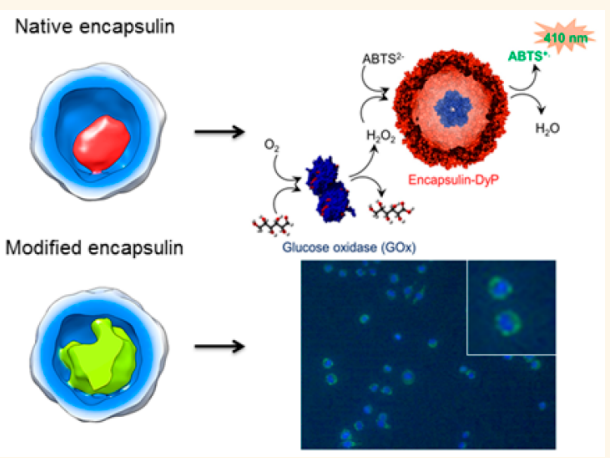
both the structure and functionality of the encapsulins allows transforming biological nanocompartments into functional systems.

KEYWORDS: encapsulin, bacterial compartments, cryo-electron microscopy, nanoreactors, nanoplatforms

$\mathrm{P}$ rotein-based nanocages have gained great interest in nanotechnology and have been developed into effective delivery agents (nanocarriers) and nanoreactors. ${ }^{1-3}$ In recent years, a class of nanocages from bacteria called the encapsulins has emerged as promising nanoplatforms, characterized by their robust nature. ${ }^{4-9}$ Nevertheless, compared to other protein-based nanocages, such as viral capsids, ${ }^{1,10}$ ferritins, ${ }^{11,12}$ and heat-shock proteins, ${ }^{13}$ the development of encapsulins for nanoplatforms is only in its infancy. ${ }^{6}$ While cages like viral capsids confine genetic material, previous studies highlighted that the encapsulins specifically confine functional and fully folded protein-based cargo, such as an enzyme or a ferritin-like protein, in that way being recognized as a bacterial semiorganelle. The encapsulin from Brevibacterium linens naturally houses a dye-decolorizing peroxidase (DyP) within its cavity, which is involved in oxidative stress. DyP is assembled as a trimer of dimers, i.e., a $240 \mathrm{kDa}$ hexamer. The specific encapsulation mechanism of DyP is mediated by its Cterminal end, which interacts specifically with a defined region of the encapsulin inner surface. We recently demonstrated that fusion of the DyP C-terminal end to a heterologous protein, such as the teal fluorescent protein (TFP), allows its packaging in these nanocontainers. ${ }^{14}$

Despite the recent sharp increase in the number of studies on encapsulins, ${ }^{4,5,7-9,14-20}$ structural characterization of encapsulins at the molecular level is yet to be explored. Encapsulins from Myxococcus xanthus (PDB entry 4PT2) ${ }^{17}$ and Pyrococcus furiosus (PDB entry 2E0Z) ${ }^{21}$ assemble into $\mathrm{T}=3$ (180-subunit, $\sim 32 \mathrm{~nm}$ diameter) nanocompartments, whereas encapsulin from Thermotoga maritima (PDB entry $3 \mathrm{DKT})^{6}$ assembles into a $\mathrm{T}=1$ (60-subunit, $24 \mathrm{~nm})$ particle. Both icosahedral particles pack ferritin-like proteins that protect cells from oxidative stress. ${ }^{6,17}$ Notably, the encapsulin fold is similar to the capsid protein fold of Hong Kong 97 (HK97)-like virions ${ }^{22}$ (including dsDNA bacteriophages and herpesviruses), which constitute

Received: October 30, 2017

Accepted: November 22, 2017

Published: November 22, 2017 

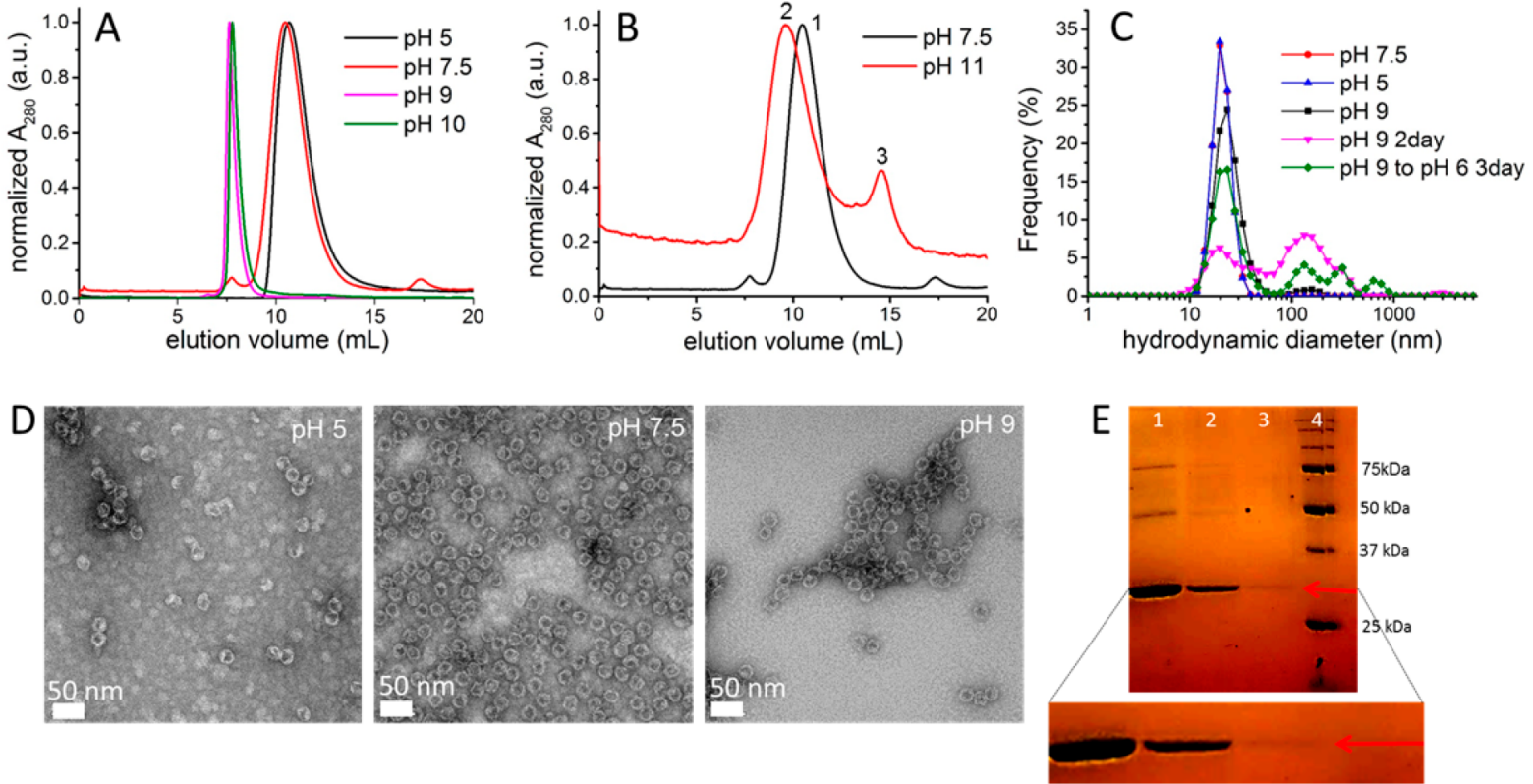

Figure 1. Characterization of encapsulin at different $\mathrm{pH}$ values. (A) Size-exclusion profiles of encapsulin at acidic, native, and basic $\mathrm{pH}$ monitored at $\lambda=280 \mathrm{~nm}$, revealing a single peak of encapsulin $(V=11-12 \mathrm{~mL}$ or $V=9 \mathrm{~mL})$ for each profile. (B) Size-exclusion profiles of encapsulin at native and highly basic $\mathrm{pH}$, showing an extra peak (number 3) at $V=15 \mathrm{~mL}$ apart from the typical encapsulin peak (numbers 1 and 2). (C) DLS-based size distributions of encapsulin at acidic, native, and basic $\mathrm{pH}$. (D) TEM images of encapsulin at acidic, native, and basic $\mathrm{pH}$, revealing intact spherical particles under all conditions. (E) Denaturing gel electrophoresis revealing the encapsulin protein band $(\sim 28 \mathrm{kDa})$ present for all samples. Lanes 1,2 , and 3 correspond to peaks 1,2 , and 3 in $\mathrm{B}$, respectively; lane 4 corresponds to the protein marker.

the most successful self-replicating system on Earth. Based on these structural similarities, encapsulins and the HK97-like capsid are hypothesized to share a common evolutionary origin; encapsulins could be of viral origin, or viruses might have originated from a similar cellular assembly. ${ }^{4,6}$ The structural characteristics at the molecular level of encapsulins that confine enzymes is still poorly understood. Deciphering the structure is crucial to advance the understanding of enzyme confinement in bacteria and the functioning of encapsulins as semiorganelles for biochemical reactions. To characterize the protein structure, as an alternative to the well-known protein crystallization, cryoelectron microscopy (cryo-EM) recently emerged as a powerful technique.

Here, we determine the structure of the encapsulin from $B$. linens with native DyP cargo (DyP-E) and foreign TFP cargo (TFP-E) using cryo-EM reconstruction. We also investigate the stability of the DyP-E system under various environmental changes to demonstrate the robust nature of the encapsulin particles. To put this robust nature into use and with the aim to substantiate experimentally that $B$. linens encapsulins are reconfigurable systems that function outside of its native environment (i.e., as bacterial semiorganelles), we separately performed an in vitro catalytic cascade involving immobilized encapsulins (using DyP-E) and an in vivo/cellular uptake study (using TFP-E). The two independent studies employing two different cargo systems, which are genetically reconstituted into $B$. linens encapsulins, demonstrate the use of encapsulins as versatile and reconfigurable nanoplatforms.

\section{RESULTS AND DISCUSSION}

Characterization of Encapsulin Stability and Structure. To investigate the robust nature of $B$. linens encapsulin, ${ }^{20}$ we characterized the stability of native DyP-E against $\mathrm{pH}$ changes and higher ionic strength. The stability of encapsulin particles in seven different $\mathrm{pH}$ values was examined (i.e., acidic condition at $\mathrm{pH} 3-5$, native condition at $\mathrm{pH} 7.5$, basic condition at $\mathrm{pH} 9-11)$. Acetate buffer was used to prepare $\mathrm{pH}$ 3 , $\mathrm{pH} 4$, and $\mathrm{pH} 5$ buffers, Tris- $\mathrm{HCl}$ buffer was used for $\mathrm{pH} 7.5$ and $\mathrm{pH} 9$ buffers, and phosphate buffer was used for $\mathrm{pH} 10$ and $\mathrm{pH} 11$ buffers. For each $\mathrm{pH}$ value, the encapsulin samples were incubated overnight and subsequently characterized using sizeexclusion chromatography (SEC), dynamic light scattering (DLS), and transmission electron microscopy (TEM), respectively. Upon mixing and overnight incubation of encapsulin in $\mathrm{pH} 3$ and $\mathrm{pH} 4$ buffers, particle aggregation was suspected to occur as the solutions became cloudy. Centrifugation of the solutions resulted in white pellets, and therefore these samples were not further analyzed.

Apart from $\mathrm{pH} 3$ and $\mathrm{pH} 4$, the encapsulin particles seemed to be stable at all tested $\mathrm{pH}$ values, which is in agreement with a previous report. ${ }^{23}$ Characterization with SEC (Figure 1A,B), DLS (Figure 1C), and TEM (Figure 1D), respectively, showed that uniform and spherical structures were present in all samples. Notably, the elution profiles from SEC were different at basic $\mathrm{pH}$ ( $\mathrm{pH} 9$ and 10); instead of eluting at $V=11-12 \mathrm{~mL}$ (characteristic for native encapsulin particles), the encapsulin incubated at higher $\mathrm{pH}$ eluted at a lower volume $(V=9 \mathrm{~mL})$, suggesting an increase in particle size, possibly due to aggregation. TEM analysis (Figure 1D) showed that typical spherical particles were still present at basic $\mathrm{pH}$, although a tendency to aggregate was observed. Aggregation of particles was clearly seen when the incubation at elevated $\mathrm{pH}$ was prolonged to 2 days (Figure 1C), which could be partially reversed by adjusting the $\mathrm{pH}$ back to around neutral. Furthermore, we also noted that a second peak at $V=15$ $\mathrm{mL}$ appeared in the case of encapsulin at $\mathrm{pH} 11$ (Figure 1B), indicating the formation of smaller particles as it eluted at a higher volume than intact particles $(V=11-12 \mathrm{~mL})$. This 
phenomenon was not observed previously at lower $\mathrm{pH}$ values. The species eluting at $V=15 \mathrm{~mL}$ was confirmed to be encapsulin-based by SDS-PAGE (Figure 1E, red arrow) since the protein band showed up at the same position as the band of native encapsulin at $\mathrm{pH}$ 7.5. Hence, it is concluded that native B. linens encapsulin partially disassembles at $\mathrm{pH} 11$. Similar particle disintegration at elevated $\mathrm{pH}$ has been reported for $\mathrm{T}$. maritima encapsulin. ${ }^{15}$

To investigate the effect of increasing ionic strength, DyP-E at native conditions $(\mathrm{pH} 7.5)$ and elevated $\mathrm{pH}(\mathrm{pH} 9)$ were incubated with either $1 \mathrm{M} \mathrm{NaCl}, 1 \mathrm{M} \mathrm{MgCl}_{2}$, or $1 \mathrm{M} \mathrm{CaCl}_{2}$ in the solution. At both $\mathrm{pH} 7.5$ and $\mathrm{pH} 9$, the encapsulin particles eluted at a similar elution volume compared to the samples with no salt added (Figure S1). This finding indicates that the increase in ionic strength (up to the tested range) does not significantly compromise the stability of encapsulin particles. This is in stark contrast, for instance, with the Cowpea Chlorotic Mottle plant virus (CCMV), which already disassembles when the ionic strength is increased to $\sim 1 \mathrm{M}$ at an elevated $\mathrm{pH}^{24}$ As for several (nano)technological applications nonaqueous conditions are required, the effect of the addition of organic solvents was examined. Here, we chose two of the most common organic solvents added to protein solutions (usually for chemical modifications), ${ }^{25,26}$ which are ethanol and DMSO. On the basis of characterization with TEM, we could still observe the presence of intact, spherical particles upon the addition of up to $40 \%$ DMSO to the encapsulin solution at $\mathrm{pH} 7.5$ (Figure S2). However, the number of intact particles was noticeably low, and most of them were already disassembled/degraded into smaller fragments, even already in the presence of $20 \%$ DMSO. Similarly, addition of $40 \%$ ethanol also resulted in predominantly degraded fragments, and only a very small amount of intact particles could be observed (Figure S2). On the contrary, addition of up to $20 \%$ ethanol did not seem to affect the structures, as they were highly comparable to the native encapsulin structures (Figure S2).

We used 3D cryo-EM to analyze the native structures of encapsulin from B. linens loaded with its natural cargo, DyP (DyP-E), or with TFP (TFP-E). Nonloaded encapsulin (nl-E) was included as a control (Figure $2 \mathrm{~A}-\mathrm{C}$ ). Whereas DyP- and TFP-loaded encapsulin particles were homogeneous, with an internal density corresponding to the cargo, nl-E showed two types of particles, either empty or containing some material in the internal cavity at a 1:5 ratio (Figure 2A, white and black arrows, respectively). The presence of material inside nl-E is attributed to random/statistical packaging of cellular components that might occur during in vivo assembly; such a phenomenon was also indicated by previously reported broad distribution of masses of nl-E particles analyzed by native mass spectrometry. ${ }^{14,20}$ A 3D reconstruction (3DR) was obtained for each of the three sets of particles (Figure 2D-F). Based on a 0.5 Fourier shell correlation (FSC) threshold, the resolution was $11.4 \AA$ for nl-E and 13.5 and $15 \AA$ for TFP-E and DyP-E, respectively. These values suggested that structural integrity was better preserved in nl-E than in cargo-loaded encapsulins, in accordance with previous biophysical analyses. ${ }^{20}$ Our biochemical analysis further confirmed that nl-E particles do not undergo partial disassembly at elevated $\mathrm{pH}$ (i.e., a tendency to aggregate was observed at basic $\mathrm{pH}$, instead of forming smaller particles, Figure S3). The outer diameter, determined from spherically averaged radial density plots of the $3 \mathrm{DR}$, was $228 \AA$ for nl-E and TFP-E, but DyP-E measured 232 Å. Despite
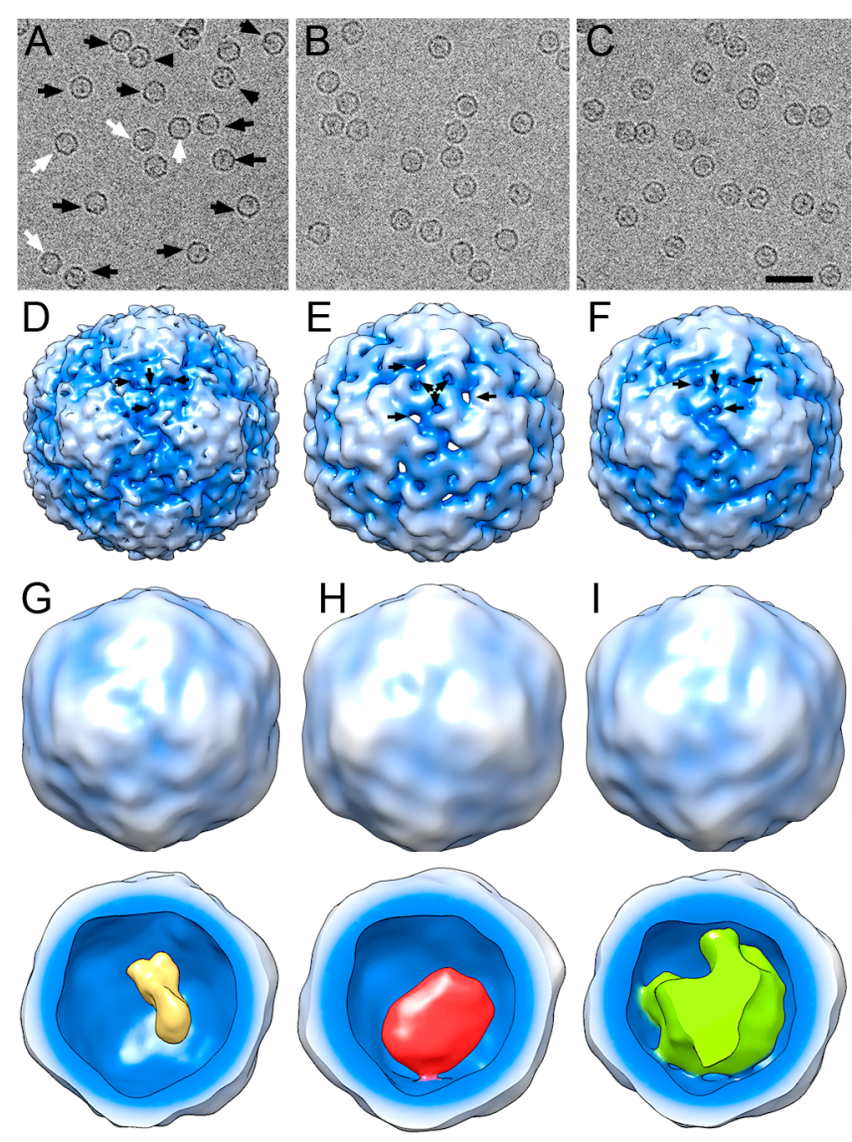

Figure 2. Cryo-EM images and 3DR of nonloaded and DyP- and TFP-loaded encapsulin from B. linens. (A-C) Cryo-electron micrographs of purified nonloaded encapsulin (nl-E) (A), DyPloaded encapsulin (DyP-E) (B), and TFP-loaded encapsulin (TFPE) (C). In A, white and black arrows indicate empty nl-E and nl-E with packed material, respectively. Bar, 500 A. (D-F) 3DR with icosahedral symmetry of nl-E (D), DyP-E (E), and TFP-E (F) viewed along a 2 -fold axis of symmetry contoured at $2 \sigma$ above the mean density. Arrows indicate pores on the outer surface. (G-I) 3DR with no symmetry of nl-E (G), DyP-E (H), and TFP-E (I). Top row, the radially color-coded outer surfaces viewed along a 2 fold axis of symmetry contoured at $2 \sigma$ above the mean density; bottom row, 3DR with the front half of the protein shell removed. Cargo is yellow (nl-E), red (DyP-E), or green (TFP-E).

this $\sim 2 \%$ size increase, the inner diameter was identical for the three maps. Encapsulin maps were icosahedral shells with an average thickness of $28-30 \AA$, and the particle is based on a T $=1$ lattice.

At the moderate resolutions achieved, some features are distinguished; the capsid is formed by 12 slightly outwardprotruding pentamers, each consisting of five curved, elongated subunits. Particles have $\sim 5-8 \AA$ diameter holes that extend through the capsid. These pores were located around the 3-fold axes (Figure $2 \mathrm{D}-\mathrm{F}$, arrows) and might serve as channels through which solutes can be interchanged with the cytoplasm. To infer cargo organization, 3DRs of DyP-E, TFP-E, and nl-E particles were calculated without imposing icosahedral symmetry to avoid smearing cargo features (Figure 2G-I). Although the three maps showed smooth topography (average resolution $\sim 24 \AA$ ), the cargo density exhibited distinct features. Whereas DyP-related density was connected to the inner surface of DyP-E at one 3-fold axis, the TFP-related density occupied a large part of the inner cavity that, depending on the 
map contour, was connected at six or 12 locations. The internal density of nl-E capsids was much smaller, $\sim 30$ and 15\% less compared to those of DyP-E and TFP-E, respectively.

Encapsulin maps for $B$. linens were similar to the map for the T. maritima $\mathrm{T}=1$ encapsulin particle, which has a smaller diameter (230-240 ̊). To evaluate this structural resemblance and considering a similar encapsulin fold, we performed docking analysis of the T. maritima encapsulin crystallographic model (PDB entry 3DKT) into the cryo-EM density maps of nonloaded and loaded $\mathrm{T}=1$ encapsulins. A total of 60 monomers of T. maritima encapsulin accounted well for the protein shell of $B$. linens nl-E (Figure 3A). This model includes

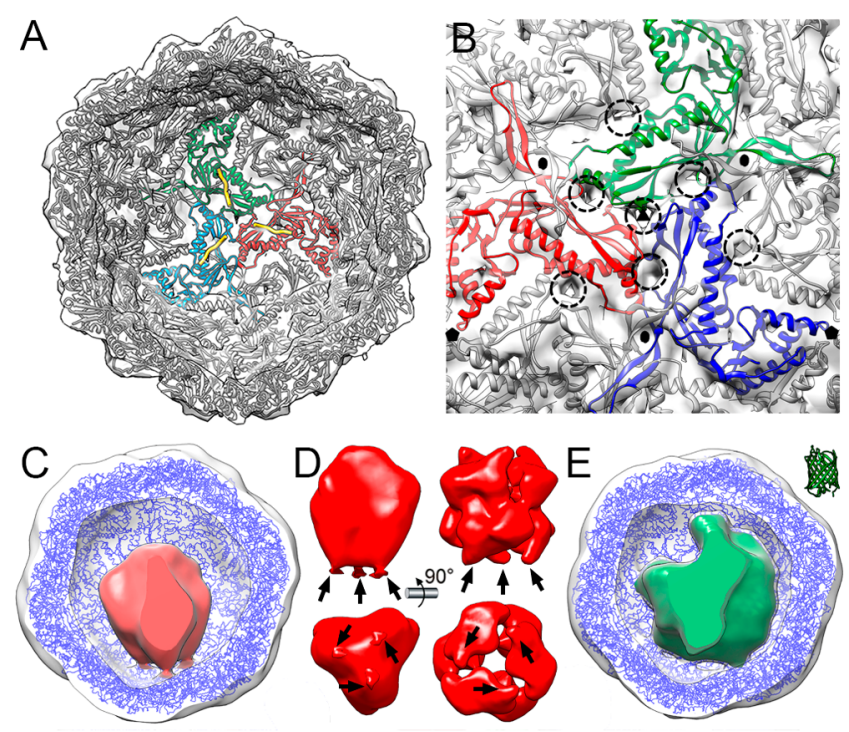

Figure 3. Pseudoatomic models of nl-E, DyP-E, and TFP-E. (A) nlE inner surface viewed along a 3-fold axis, with docked T. maritima encapsulin atomic coordinates. Encapsulin monomers at the 3-fold axis are depicted in red, green, and blue, and their corresponding Flp C-terminal ends in yellow. (B) nl-E outer surface viewed along a 3-fold axis. There are numerous pores at and around the 3-fold axis (dashed circles). The cargo density has been removed computationally. Black symbols indicate icosahedral symmetry axes. (C) DyP-E map with $C_{3}$ symmetry from inside, with docked $T$. maritima encapsulin (blue) and DyP density (red). (D) DyP cryoEM density extracted from the map shown in $\mathrm{C}$; arrows indicate connections to the inner encapsulin surface (left). DyP density calculated from negative staining electron microscopy ${ }^{6}$ (right). (E) TFP-E map from inside, with docked T. maritima encapsulin (blue) and TFP density (green). The TFP atomic structure (2HQK) is shown at the same scale (top right).

the C-terminal extension of the packaged protein, a ferritin-like protein (Flp) from T. maritima (Figure 3A, yellow). In addition, most pores in the T. maritima encapsulin shell colocalized with the pores in B. linens nl-E (Figure 3B, dashed circles). In the functional context of native DyP-E, we were able to pinpoint the location of both the encapsulated DyP (i.e., connected to one of the 3-fold axes) and the pores (i.e., mostly at the 3-fold axes); we could deduce that the pores at the corresponding 3-fold axis can function as direct gates for substrates from the cytoplasm to access the confined enzyme. Together with previous results from the analyses on particle stability, these findings indicate that the encapsulin acts as a robust shell encasing the confined protein cargo, while at the same time allowing molecular flux through the shell resulting from the porous structure.
The role of the DyP C-terminal extension is, as it is for Flp, to target the cargo protein to the binding sites on the encapsulin interior. Considering that DyP is organized as a trimer of dimers, we calculated a 3DR of DyP-E with $C_{3}$ symmetry (Figure 3C). The map resolution was slightly improved compared to the map calculated with no symmetry (25 Å versus $28 \AA$ ). Furthermore, DyP was solved as a density with three connections that match with the location of the Flp C-terminal extensions (Figure 3D, left). The B. linens DyP, expressed in the absence of encapsulin, was used to calculate a map from negatively stained particles (Figure 3D, right, EMD entry 1530). ${ }^{6}$ This recombinantly expressed DyP is similar to our native encapsulated DyP model. The cryo-EM hexameric DyP map highlighted the structural differences between the two trimers; the trimer connected to the encapsulin capsid interior probably has ordered C-terminal extensions, whereas the other three C-terminal extensions are probably disordered. The cryoEM-packed hexameric DyP was observed as an elongated density $\left(\sim 114 \AA\right.$ long $\times \sim 92 \AA$ wide; $\sim 3.3 \times 10^{5} \AA^{3}$ volume $)$, compatible with the dimensions and volume of a recombinant hexameric DyP $\left(\sim 104 \times 104 \AA ; 2.85 \times 10^{5} \AA^{3}\right)$. The cryo-EM TFP-related density was $\sim 5.1 \times 10^{5} \AA^{3}$ (Figure $3 \mathrm{E}$ ); a TFP monomer (PDB entry $2 \mathrm{HQK}$; a TFP derived from Clavularia sp. $)^{27}$ is $4.7 \times 10^{4} \AA^{3}$, which suggested that 10-12 TFP copies/ particle are encapsulated, compatible with previous mass spectrometry analysis. ${ }^{14}$ The internal volume of the $B$. linens encapsulin particle $\left(\sim 2.6 \times 10^{6} \AA^{3}\right)$ does not limit loading of the natural cargo, a single hexameric DyP (240 kDa). DyP loading capacity is instead constrained by the shape and oligomeric state of the cargo enzyme. Although the DyP hexamer has six C-terminal extensions that are able to interact with their cargo-binding sites, its quaternary structure indicates that only three of these extensions are bound. By similar reasoning, our TFP-E map indicates that the TFP monomers are evenly distributed in the encapsulin interior, and the TFP-E particle can contain $10-12$ copies $(\sim 400 \mathrm{kDa})$ of monomeric TFP.

DyP-E and TFP-E have lower breaking force than nl-E, according to atomic force microscopy (AFM) studies, which implies that DyP- and TFP-loaded encapsulins are less stable. ${ }^{20}$ The estimated resolution values for the calculated maps, which are inversely related to the number of particles in each map, also suggested that structural integrity was better preserved in $\mathrm{nl}$-E than in cargo-loaded encapsulins. As suggested, ${ }^{20}$ binding of the three cargo-anchoring sequences to the shell interior could distort locally icosahedral symmetry for DyP-E. This local distortion in a 3-fold symmetry axis might be needed for optimal function of the hexameric DyP enzyme complex. For TFP-E, binding of multiple TFP (up to 10-12 copies) to the interior surface of the encapsulin pocket results in a similar overall destabilization of the shell.

In Vitro Study of Encapsulin as Nanoreactors on the Surface. To demonstrate the functionality of encapsulins for in vitro applications, we investigated the performance of native DyP-E as a bionanoreactor. The confined DyP inside the encapsulin cavity allows the particles to catalytically oxidize dye molecules, for example 2,2' -azinobis(3-ethylbenzothiazoline-6sulfonic acid) (ABTS) dye, while reducing hydrogen peroxide to water. ${ }^{19,28} \mathrm{We}$ immobilized the encapsulin particles on a glass surface in order to ease the particle recovery as well as to demonstrate the catalysis on a surface, which is commonly preferred for in vitro application such as protein-based sensors. $^{29,30}$ In comparison to tethering enzymes directly 
A

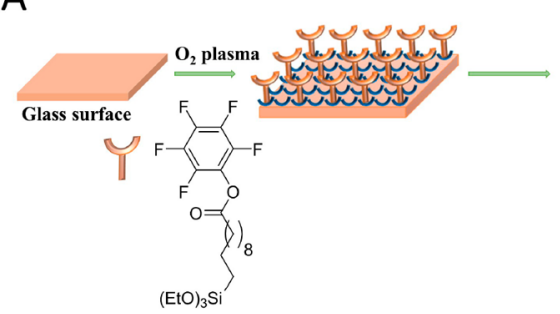

C

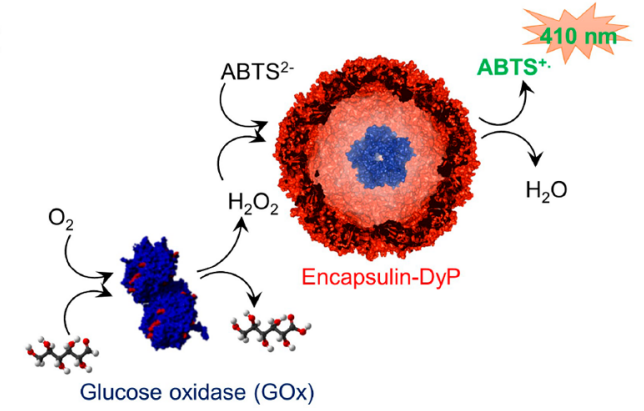

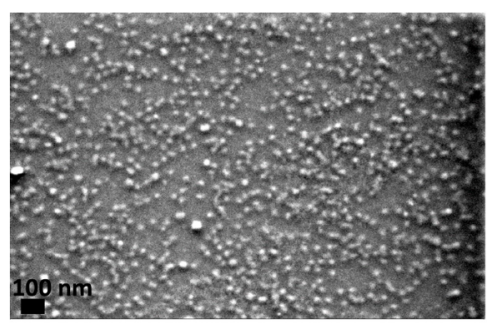

D

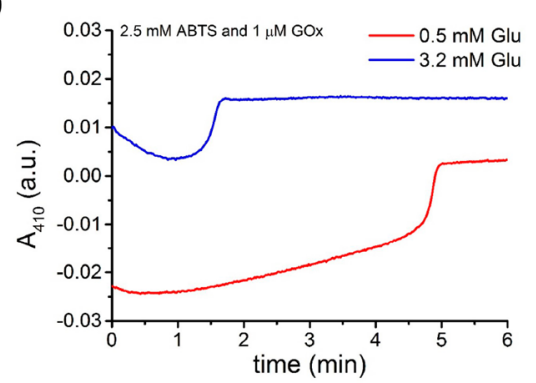

Figure 4. Surface-immobilized encapsulin as a bionanoreactor. (A) Immobilization strategy of encapsulin particles on a glass surface using PFPS molecules as linkers. (B) SEM image of surface-immobilized encapsulin showing the presence of nanometer-sized particles ( 24 nm) on the surface. (C) Schematic representation of a tandem system consisting of GOx and DyP-E. The initial substrate glucose is oxidized by GOx while producing $\mathrm{H}_{2} \mathrm{O}_{2}$, which in turn is converted by DyP into water while producing green-colored ABTS radical. (D) Catalytic assay of the tandem system monitored at $\lambda=410 \mathrm{~nm}$ based on the absorption of the ABTS radical cation.

onto the surface, the immobilized encapsulin would offer a robust and chemically modifiable shell for, in principle, a variety of confined enzymes. The glass surface was first modified with pentafluorophenyl silicate (Figure 4A), which introduces surface reactivity and allows chemical reaction with lysine residues present on the encapsulin surface. ${ }^{31}$ We have previously reported that the lysine residues of $B$. linens encapsulin are indeed available for chemical modification. ${ }^{18}$

To confirm that the particles were covalently immobilized onto the surface using this approach, we performed a contact angle measurement on the encapsulin-modified glass surface, which resulted in the contact angle of $55-60^{\circ}$ as opposed to $75-85^{\circ}$ for a PFPS-modified glass surface (before adding the encapsulin). The decrease in the contact angle after encapsulin addition indicated a successful immobilization, as the presence of encapsulin changed the surface properties into a more polar state (in comparison to the surface with PFPS only). Furthermore, as shown in Figure 4B, we were also able to visualize the encapsulin particles on the surface using scanning electron microscopy (SEM). On the basis of multiple images recorded with SEM, we estimated the percentage of coverage area to be $35 \pm 5 \%$ with up to $1.16 \times 10^{11}$ encapsulin particles immobilized on the surface (area: $1 \times 1.5 \mathrm{~cm}^{2}$ ).

After surface attachment, we investigated the catalytic activity by carrying out a cascade reaction on the surface. The encapsulin enzymatic activity was tested based on the oxidization of ABTS dye while reducing $\mathrm{H}_{2} \mathrm{O}_{2}$ produced by an additional enzyme called glucose oxidase ( $\mathrm{GOx}$ ) that was added into the solution (Figure 4C). We chose the well-studied tandem GOx-peroxidase system, as it has been proven useful as a biosensor for glucose detection, ${ }^{28,32,33}$ thus providing our system with a possible functionality. The oxidation of ABTS into its green-colored radical form was monitored at $\lambda=410$ $\mathrm{nm}$ for up to $6 \mathrm{~min}$ (Figure 4D) at different glucose concentrations. In our experiments, GOx $(1 \mu \mathrm{M})$ was added as the final compound into the reaction mixture, as it kick-starts the entire cascade reaction. Noticeably, the DyP-catalyzed formation of ABTS-based radical did not start immediately after GOx addition $(t=0$, Figure $4 \mathrm{D})$, which is attributed to GOxcatalyzed $\mathrm{H}_{2} \mathrm{O}_{2}$ production and diffusion to the surfaceimmobilized, encapsulated DyP. As expected, this "lag phase" was shortened by increasing the concentration of glucose as more $\mathrm{H}_{2} \mathrm{O}_{2}$ is produced with increasing glucose concentration. In a similar way the "lag phase" decreased when the surfaceimmobilized encapsulin was added into a premixed glucoseGOx solution with $\mathrm{H}_{2} \mathrm{O}_{2}$ already present (Figure S4), which did not change the reaction rate.

On the basis of the maximum slope $\left(\max \mathrm{d} A_{410} / \mathrm{d} t\right)$, we calculated the reaction rates (i.e., the rate of the ABTS radical cation being formed) to be $2.67 \mu \mathrm{M} / \mathrm{min}$ for $0.5 \mathrm{mM}$ glucose, $1.57 \mu \mathrm{M} / \mathrm{min}$ for $3.2 \mathrm{mM}$ glucose, and $1.83 \mu \mathrm{M} / \mathrm{min}$ for $5 \mathrm{mM}$ glucose. As the activity at lower concentrations is minimal (see Supporting Information section S4), we assume that $0.5 \mathrm{mM}$ glucose was sufficient to achieve the maximum reaction rate on the surface (i.e., $V_{\max }=2.67 \mu \mathrm{M} / \mathrm{min}$ ), particularly since increasing glucose concentrations did not lead to a higher reaction rate and likely resulted in substrate inhibition instead. ${ }^{34}$ Considering that the number of the encapsulins immobilized is equal to the number of confined DyP since each particle encapsulates one DyP (i.e., $1.16 \times 10^{11}$ particles), we could calculate the apparent turnover number of $\left(V_{\max } / \mathrm{DyP}\right.$ concentration) to be $k_{\text {cat }}=115.4 \mathrm{~s}^{-1}$ (within the reaction volume of $500 \mu \mathrm{L}$, the total protein concentration $=3.86 \times$ $\left.10^{-10} \mathrm{M}\right)$. The obtained turnover number is comparable in magnitude to reported turnover numbers for (nonencapsulated) DyP in the literature (i.e., $k_{\text {cat }}=224 \mathrm{~s}^{-1}$ ), ${ }^{35}$ indicating that the surface immobilization did not lead to a significant activity loss. We have demonstrated here the catalytic activity of surface-immobilized encapsulin in vitro and the use of $B$. linens encapsulins as bionanoreactors.

Cellular Uptake of Encapsulin. To evaluate the use of $B$. linens encapsulins for in vivo applications, we investigated the 

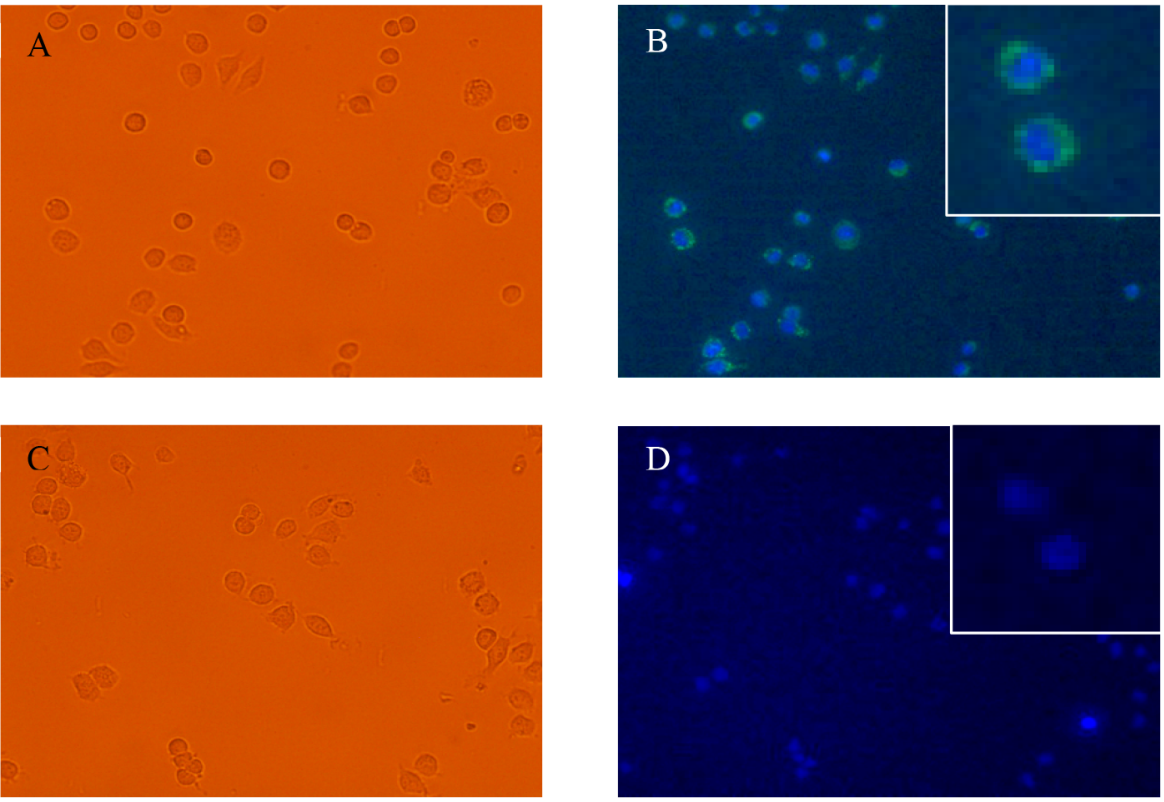

Figure 5. Fluorescence microscopy images of J774 macrophages treated with TFP-E. (A) Bright-field image of macrophages treated with 0.033 $\mu \mathrm{M}$ TFP-E. (B) Fluorescence microscopy images of macrophages treated with $0.033 \mu \mathrm{M}$ TFP-E showing fluorescence from TFP cargo protein taken up by the cells. The TFP (green-colored) does not enter the nucleus (inset). (C) Bright-field image of the negative control where macrophages were treated with PBS only. (D) Fluorescence microscopy images showing no green fluorescence in cells treated with PBS only.

uptake of the encapsulin with a fluorescent protein cargo (TFPE) by mammalian J774 macrophages. A more detailed biochemical analyses of TFP-E particles has been reported in previous work by our group. ${ }^{14}$ Notably, the modified particles remain intact and stable over five years of storage at $4{ }^{\circ} \mathrm{C}$ (Figure S5), further confirming their robust nature even with a non-native cargo.

After the addition of TFP-E to the macrophage cells and subsequent incubation for $4 \mathrm{~h}$, images were taken with a fluorescent microscope to visualize the TFP cargo (excitation at $\lambda=460-490 \mathrm{~nm}$, emission at $\lambda=525 \mathrm{~nm}$ ) and the nuclei of the cells with Hoechst-staining (excitation at $\lambda=360-370 \mathrm{~nm}$, emission at $\lambda=460 \mathrm{~nm}$ ) (Figure 5). The negative control, a sample of cells that were not treated with any encapsulin particles and that remain present after the removal of culture medium, does not show any fluorescence signal (Figure 5C,D). For $0.033 \mu \mathrm{M}$ TFP-E, the treated macrophages show a high fluorescent signal as shown in Figure 5A,B. The green-colored signal that is found around the nucleus indicates that the TFP cargo was taken up by the macrophages. On the basis of the fluorescence signal from the cells (Figure 5B, inset), TFP-E appears to enter the macrophages effectively, but does not enter the nucleus.

This study shows that the encapsulins can be used to confine a foreign protein cargo and preserve their structural integrity for a prolonged time in vitro, and the cargo can be taken up by mammalian cells. Further studies are needed to identify whether the spherical particles stay intact inside the cells as well as the uptake mechanism.

\section{CONCLUSION}

We have provided structural insight into the encapsulins from B. linens using cryo-EM, confirming that the native icosahedral, cage-like assemblies confine a single peroxidase $\mathrm{DyP}$ within its cavity. Together with particle stability analyses, our findings indicate the encapsulin acts as a robust shell protecting the confined DyP, while at the same time allowing molecular flux through the shell due to the porous structure. Using the same analysis, we also characterized the structure of modified encapsulin particles with non-native cargo (i.e., a fluorescent protein). Compared to the empty encapsulin (nl-E) the protein cages that contain a cargo are slightly larger, which is in line with our previous analysis. ${ }^{20}$ The data further confirm the directive role the $\mathrm{C}$-terminal fragments on the cargo proteins play in their inclusion, ${ }^{6,14}$ allowing the design and preparation of encapsulin containers with a variety of guest systems.

To investigate the functionality of encapsulins as a promising agent for nanoplatforms, we demonstrated a tandem in vitro catalysis, proving that the encapsulin is able to play a role as bionanoreactors on the surface as well as the in vivo cellular uptake of modified encapsulins by mammalian macrophages. Our results provide both a functional and structural basis of $B$. linens encapsulins for the development of encapsulins as functional assemblies for nanotechnology, for instance as therapeutic agents and biosensors.

\section{METHODS}

Materials. B. linens encapsulin was recombinantly expressed in $E$. coli and purified based on reported procedures. ${ }^{14,18}$ Encapsulin samples were stored in "encapsulin storage buffer" containing 20 $\mathrm{mM}$ Tris- $\mathrm{HCl}, 150 \mathrm{mM} \mathrm{NH}_{4} \mathrm{Cl}$, and $1 \mathrm{mM} \beta$-mercaptoethanol at $\mathrm{pH}$ 7.5 at $4{ }^{\circ} \mathrm{C}$. For $\mathrm{pH}$ variation studies, $100 \mathrm{mM}$ acetate buffer $\left(\mathrm{CH}_{3} \mathrm{COONa}-\mathrm{CH}_{3} \mathrm{COOH}\right)$ was used to prepare $\mathrm{pH} 3, \mathrm{pH} 4$, and $\mathrm{pH}$ 5 buffers, $20 \mathrm{mM}$ Tris- $\mathrm{HCl}$ buffer was used for $\mathrm{pH} 7.5$ and $\mathrm{pH} 9$ buffers, and $10 \mathrm{mM}$ phosphate buffer $\left(\mathrm{Na}_{2} \mathrm{HPO}_{4}-\mathrm{NaOH}\right)$ was used for $\mathrm{pH} 10$ and $\mathrm{pH} 11$ buffers. All chemicals were purchased from Sigma-Aldrich unless stated otherwise. All incubations and reactions were conducted at room temperature unless stated otherwise.

Cryo-electron Microscopy and Image Processing. Nonloaded and DyP- and TFP-loaded B. linens encapsulin $(5 \mu \mathrm{L})$ were applied to one side of Quantifoil R 2/2 holey grids, blotted, and plunged into liquid ethane in a Leica EM CPC cryofixation unit. Samples were analyzed in a Tecnai G2 electron microscope equipped with a field emission gun operating at $200 \mathrm{kV}$, and images were recorded under 
low-dose conditions with a FEI Eagle CCD at a detector magnification of $69444 \times(2.16 \AA$ /pixel sampling rate). Image processing operations were performed using Xmipp ${ }^{36}$ and Relion ${ }^{37}$ packages integrated in the Scipion platform. ${ }^{38}$ Graphic representations were produced by UCSF Chimera. ${ }^{39}$ The Xmipp automatic picking routine was used to select 6785,19591 , and 29680 individual particle images of nonloaded and DyP- and TFP-loaded encapsulin, respectively. A 1.2-4.1 $\mu \mathrm{m}$ defocus range was determined for each image with CTFfind $4 .^{40}$ Particle images were extracted, normalized, and downsampled to a factor of 2 , with a final sampling ratio of $4.32 \AA /$ pixel. Using the Relion routine, a two-dimensional (2D) classification was performed to discard low-quality particles, and 5357, 15941, and 19779 isometric particles were selected for nonloaded and DyP- and TFP-loaded encapsulin, respectively. A 3D classification was run using the structure of Thermotoga maritima encapsulin (PDB entry 3DKT), low-pass filtered to $40 \AA$, as an initial model. When icosahedral symmetry was imposed, a single class with 4246, 12 833, and 15976 particles was obtained for nonloaded and DyP- and TFP-loaded encapsulin, respectively. When assuming no symmetry for nonloaded and DyP- and TFP-loaded encapsulin, three classes were obtained, but no significant differences were observed between them at the resolutions achieved, and particles were refined together. DyP-loaded encapsulin particles were analyzed considering $C_{3}$ symmetry, and a single class with 9930 particles was selected. These data sets were used to obtain the final 3DR using the Relion autorefinement routine. Resolutions of 3D with icosahedral symmetry were estimated from two independent half data sets using the 0.5 (or 0.3 ) criterion of the Fourier shell correlation, and the values for nonloaded and DyP- and TFP-loaded encapsulin were 11.4 (10.7), 15.1 (13.5), and 13.5 (12) $\AA$, respectively. Similarly, resolution values for asymmetric 3DR were 27.3 (23.8), 28.3 (25), and 24.2 (22.7) A. For the DyP-loaded encapsulin with $C_{3}$ symmetry, resolution was 24.7 (22.8) A. The Chimera fitting tool was used to dock the atomic crystallographic model T. maritima encapsulin into our cryo-EM encapsulin maps. The encapsulin 3DR are deposited in the Electron Microscopy Data Bank (http://www.ebi. ac.uk/pdbe/emdb) with accession no. EMD-3608 (nl-E), EMD-3612 (DyP-E), and EMD-3615 (TFP-E) for maps with icosahedral symmetry; EMD-3609 (nl-E), EMD-3614 (DyP-E) and EMD-3616 (TFP-E) for maps without imposing icosahedral symmetry; and EMD3613 (DyP-E with C3 symmetry).

Characterization of Encapsulin Stability. Size-exclusion profiles of encapsulins upon $\mathrm{pH}$ and ionic strength variation were obtained by injecting and running $500 \mu \mathrm{L}$ of each sample $(\sim 15 \mu \mathrm{M})$ into a Superose 6 preparative column 10/100 GL (GE Healthcare FPLC Äkta purifier 900 with a $24 \mathrm{~mL}$ bed volume). The size-exclusion analysis was repeated two times. The hydrodynamic size distribution of the particles was determined using a Nanotrac Wave (Microtrac) particle analyzer. For particle imaging with TEM, $5 \mu \mathrm{L}$ of a sample was applied onto Formvar-carbon-coated grids, and the liquid was drained after $30 \mathrm{~s}$. Afterward, $5 \mu \mathrm{L}$ of a staining solution consisting of uranyl acetate $(1 \% \mathrm{w} / \mathrm{v})$ was added onto the grids, and the liquid was drained after $1 \mathrm{~min}$. For protein characterization with denaturing gel electrophoresis (SDS-PAGE), experiments using 12\% polyacrylamide gel were conducted based on procedures in the literature, ${ }^{41}$ and BioSafe Coomassie (Bio-Rad) was used for visualization of protein bands.

Immobilization of Encapsulin onto a Glass/Si Surface. Substrates were rinsed with water, activated by immersion/cycling piranha solution $\left(\mathrm{H}_{2} \mathrm{SO}_{4}-\mathrm{H}_{2} \mathrm{O}_{2}, 3: 1\right)$, rinsed with water and ethanol, and then dried with a steam of nitrogen. Perfluorophenyl-11(triethoxysilyl)undecanoate (PFPS) was deposited by substrate immersion into a PFPS solution (dichloromethane (DCM), 10 $\mathrm{mM}$ ) for $24 \mathrm{~h}$ at room temperature under an argon atmosphere. The glass wafer was rinsed with DCM to remove unreacted reagent and dried in a stream of $\mathrm{N}_{2}$. Encapsulin particles were deposited from buffered solution (0.2 M phosphate buffer, $\mathrm{pH}$ 7.2). The particles were drop-coated on the flat glass substrate overnight in a closed vessel to avoid solvent evaporation. Afterward, the glass was rinsed with buffer solution to remove nonimmobilized particles.

Catalysis of Surface-Immobilized Encapsulin. A $200 \mu \mathrm{L}$ amount of glucose (three different final concentrations: $0.5,3.2$, and
$5 \mathrm{mM})$ and $200 \mu \mathrm{L}$ of ABTS $(2.5 \mathrm{mM})$ in Tris-HCl buffer $\mathrm{pH} 7.5$ were placed inside a cuvette together with the glass surface with the immobilized encapsulin particles. Afterward, $100 \mu \mathrm{L}$ of GOx $(1 \mu \mathrm{M})$ in PBS buffer $\mathrm{pH} 7.4$ was added into the cuvette to start the reaction, and the absorption value at $\lambda=410 \mathrm{~nm}$ was immediately recorded for at least 6 min using a PerkinElmer Lamba 850 UV-visible spectrometer. As control experiments, a PFPS-modified glass surface without encapsulin particles was used for similar kinetic studies.

The reaction rate based on radical ABTS production is calculated as follows:

$$
\text { reaction rate }=\frac{\mathrm{d}[\text { radical ABTS }]}{\mathrm{d} t}=\frac{\mathrm{d} A}{\mathrm{~d} t} \frac{1}{\varepsilon x b}
$$

where $A$ is the absorbance of radical ABTS at $\lambda=410 \mathrm{~nm}, \varepsilon$ is the extinction coefficient of radical ABTS at $\lambda=410 \mathrm{~nm}\left(36000 \mathrm{M}^{-1}\right.$ $\left.\mathrm{cm}^{-1}\right)$, and $b$ is the cuvette path length $(1 \mathrm{~cm}) . \mathrm{d} A / \mathrm{d} t$ corresponds to the maximum slope of the kinetic plots in Figures 4D and S4 derived using OriginPro 9.0 software. The apparent turnover number $k_{\text {cat }}$ was calculated based on $V_{\max } /$ DyP concentration. Although the particles were immobilized on the glass surface, the catalytic assay was performed in solution (i.e., the modified surface was fully immersed inside a cuvette). The DyP concentration was calculated based on the number of particles on the surface and the volume of the reaction. The catalytic assay was repeated at least two times.

Cell Experiments. B. linens encapsulins containing mTFP (monomeric teal fluorescent protein) were recombinantly produced in $E$. coli and purified using the same protocol established for native $B$. linens encapsulin. The concentration of TFP-E was $0.30 \mu \mathrm{M}$. Murine macrophage cells (J774) were cultivated in DMEM. Following the cultivation, $100 \mu \mathrm{L}$ of $\sim 2000$ cells was plated per well on a 96-well plate, and either 0 or $10 \mu \mathrm{L}$ of TFP-E was added into the well ( 0 or $0.03 \mu \mathrm{M}$ TFP-E, respectively). The treated cells were incubated at 37 ${ }^{\circ} \mathrm{C}$ and $5 \% \mathrm{CO}_{2}$ for $4 \mathrm{~h}$ to allow the cells to take up the particles. After $3.5 \mathrm{~h}$ of incubation, Hoechst nucleus stain was added to a final concentration of $0.5 \mu \mathrm{g} / \mathrm{mL}$. Following the incubation, the medium was removed and the cells were rinsed $2 \times$ with phosphate buffer saline (10 mM PBS, pH 7.4) to further remove nonabsorbed species. A 100 $\mu \mathrm{L}$ amount of HEPES was added to the cells prior to visualization. For the visualization based on fluorescence, the cells were imaged using a fluorescence microscope (Olympus TH4-200 with an X-Cite series $120 \mathrm{pc} Q$ laser from Lumen Dynamics, excitation at $\lambda=460-490 \mathrm{~nm}$, emission at $\lambda=525 \mathrm{~nm}$ ).

Data and Schematic Representation. All data plotting and mathematical calculations were performed with OriginPro 9.0 software. Protein structures are rendered using PyMOL 1.3 software, and chemical structures are drawn using ChemBioDraw Ultra 12.0 software.

\section{ASSOCIATED CONTENT}

\section{Supporting Information}

The Supporting Information is available free of charge on the ACS Publications website at DOI: 10.1021/acsnano.7b07669.

Native encapsulin (DyP-E) response to variation of ionic strength and addition of organic solvents, analysis of empty encapsulins (nl-E), cascade catalysis of GOx and surface-immobilized DyP-E, and stability of modified encapsulin (TFP-E) over time (PDF)

\section{AUTHOR INFORMATION}

\section{Corresponding Authors}

*E-mail: jrcaston@cnb.csic.es.

*E-mail: j.j.l.m.cornelissen@utwente.nl. ORCID

Jeroen J. L. M. Cornelissen: 0000-0002-9728-5043 Notes

The authors declare no competing financial interest. 


\section{ACKNOWLEDGMENTS}

We acknowledge financial support from the ERC Consolidator Grant (Protcage) and the Indonesia Endowment Fund for Education (LPDP). This work was supported in part by grants from the Spanish Ministry of Economy and Competitivity (BFU2014-55475 to J.R.C.) and the Comunidad Autónoma de Madrid (S2013/MIT-2807 to J.R.C.). We are grateful to Dr. E. G. Keim, M. A. Smithers, and R. van der Hee (MESA+ Institute for Nanotechnology, University of Twente) for assistance with TEM, SEM imaging, and cell culture. We are grateful to Prof. N. Ban and Dr. M. Sutter for the kind donation of plasmids and to Dr. J. Snijder for fruitful discussions.

\section{REFERENCES}

(1) Uchida, M.; Klem, M. T.; Allen, M.; Suci, P.; Flenniken, M.; Gillitzer, E.; Varpness, Z.; Liepold, L. O.; Young, M.; Douglas, T. Biological Containers: Protein Cages as Multifunctional Nanoplatforms. Adv. Mater. 2007, 19, 1025-1042.

(2) Peek, L. J.; Middaugh, C. R.; Berkland, C. Nanotechnology in Vaccine Delivery. Adv. Drug Delivery Rev. 2008, 60, 915-928.

(3) Lee, E. J.; Lee, N. K.; Kim, I. S. Bioengineered Protein-Based Nanocage for Drug Delivery. Adv. Drug Delivery Rev. 2016, 106, 157171.

(4) Giessen, T. W. Encapsulins: Microbial Nanocompartments with Applications in Biomedicine, Nanobiotechnology and Materials Science. Curr. Opin. Chem. Biol. 2016, 34, 1-10.

(5) Choi, B.; Moon, H.; Hong, S. J.; Shin, C.; Do, Y.; Ryu, S.; Kang, S. Effective Delivery of Antigen-Encapsulin Nanoparticle Fusions to Dendritic Cells Leads to Antigen-Specific Cytotoxic T Cell Activation and Tumor Rejection. ACS Nano 2016, 10, 7339-7350.

(6) Sutter, M.; Boehringer, D.; Gutmann, S.; Guenther, S.; Prangishvili, D.; Loessner, M. J.; Stetter, K. O.; Weber-Ban, E.; Ban, N. Structural Basis of Enzyme Encapsulation into a Bacterial Nanocompartment. Nat. Struct. Mol. Biol. 2008, 15, 939-947.

(7) Moon, H.; Lee, J.; Min, J.; Kang, S. Developing Genetically Engineered Encapsulin Protein Cage Nanoparticles as a Targeted Delivery Nanoplatform. Biomacromolecules 2014, 15, 3794-3801.

(8) Tamura, A.; Fukutani, Y.; Takami, T.; Fujii, M.; Nakaguchi, Y.; Murakami, Y.; Noguchi, K.; Yohda, M.; Odaka, M. Packaging Guest Proteins into the Encapsulin Nanocompartment from Rhodococcus erythropolis N771. Biotechnol. Bioeng. 2015, 112, 13-20.

(9) Corchero, J. L.; Cedano, J. Self-Assembling, Protein-Based Intracellular Bacterial Organelles: Emerging Vehicles for Encapsulating, Targeting and Delivering Therapeutical Cargoes. Microb. Cell Fact. 2011, 10, 92.

(10) Kushnir, N.; Streatfield, S. J.; Yusibov, V. Virus-Like Particles as a Highly Efficient Vaccine Platform: Diversity of Targets and Production Systems and Advances in Clinical Development. Vaccine 2012, 31, 58-83.

(11) Zhen, Z.; Tang, W.; Todd, T.; Xie, J. Ferritins as Nanoplatforms for Imaging and Drug Delivery. Expert Opin. Drug Delivery 2014, 11, 1913-1922.

(12) Zeth, K.; Hoiczyk, E.; Okuda, M. Ferroxidase-Mediated Iron Oxide Biomineralization: Novel Pathways to Multifunctional Nanoparticles. Trends Biochem. Sci. 2016, 41, 190-203.

(13) Choi, S. H.; Kwon, I. C.; Hwang, K. Y.; Kim, I. S.; Ahn, H. J. Small Heat Shock Protein as a Multifunctional Scaffold: Integrated Tumor Targeting and Caspase Imaging within a Single Cage. Biomacromolecules 2011, 12, 3099-3106.

(14) Rurup, W. F.; Snijder, J.; Koay, M. S. T.; Heck, A. J. R.; Cornelissen, J. J. L. M. Self-Sorting of Foreign Proteins in a Bacterial Nanocompartment. J. Am. Chem. Soc. 2014, 136, 3828-3832.

(15) Cassidy-Amstutz, C.; Oltrogge, L.; Going, C. C.; Lee, A.; Teng, P.; Quintanilla, D.; East-Seletsky, A.; Williams, E. R; Savage, D. F. Identification of a Minimal Peptide Tag for In Vivo and In Vitro Loading of Encapsulin. Biochemistry 2016, 55, 3461-3468.
(16) Contreras, H.; Joens, M. S.; McMath, L. M.; Le, V. P.; Tullius, M. V.; Kimmey, J. M.; Bionghi, N.; Horwitz, M. A.; Fitzpatrick, J. A.; Goulding, C. W. Characterization of a Mycobacterium tuberculosis Nanocompartment and Its Potential Cargo Proteins. J. Biol. Chem. 2014, 289, 18279-18289.

(17) McHugh, C. A.; Fontana, J.; Nemecek, D.; Cheng, N. Q.; Aksyuk, A. A.; Heymann, J. B.; Winkler, D. C.; Lam, A. S.; Wall, J. S.; Steven, A. C.; et al. A Virus Capsid-Like Nanocompartment that Stores Iron and Protects Bacteria from Oxidative Stress. EMBO J. 2014, 33, 1896-1911.

(18) Putri, R. M.; Fredy, J. W.; Cornelissen, J. J. L. M.; Koay, M. S. T.; Katsonis, N. Labelling Bacterial Nanocages with Photo-Switchable Fluorophores. ChemPhysChem 2016, 17, 1815-1818.

(19) Rahmanpour, R.; Bugg, T. D. H. Assembly In Vitro of Rhodococcus jostii RHAl Encapsulin and Peroxidase DypB to Form a Nanocompartment. FEBS J. 2013, 280, 2097-2104.

(20) Snijder, J.; Kononova, O.; Barbu, I. M.; Uetrecht, C.; Rurup, W. F.; Burnley, R. J.; Koay, M. S. T.; Cornelissen, J. J. L. M.; Roos, W. H.; Barsegov, V.; et al. Assembly and Mechanical Properties of the CargoFree and Cargo-Loaded Bacterial Nanocompartment Encapsulin. Biomacromolecules 2016, 17, 2522-2529.

(21) Akita, F.; Chong, K. T.; Tanaka, H.; Yamashita, E.; Miyazaki, N.; Nakaishi, Y.; Suzuki, M.; Namba, K.; Ono, Y.; Tsukihara, T.; et al. The Crystal Structure of a Virus-Like Particle from the Hyperthermophilic Archaeon Pyrococcus furiosus Provides Insight into the Evolution of Viruses. J. Mol. Biol. 2007, 368, 1469-1483.

(22) Abrescia, N. G.; Bamford, D. H.; Grimes, J. M.; Stuart, D. I. Structure Unifies the Viral Universe. Annu. Rev. Biochem. 2012, 81, $795-822$.

(23) Valdes-Stauber, N.; Scherer, S. Isolation and Characterization of Linocin M18, a Bacteriocin Produced by Brevibacterium linens. Appl. Environ. Microbiol. 1994, 60, 3809-3814.

(24) Lavelle, L.; Michel, J. P.; Gingery, M. The Disassembly, Reassembly and Stability of CCMV Protein Capsids. J. Virol. Methods 2007, 146, 311-316.

(25) Teramoto, H.; Nakajima, K.; Takabayashi, C. Chemical Modification of Silk Sericin in Lithium Chloride/Dimethyl Sulfoxide Solvent with 4-Cyanophenyl Isocyanate. Biomacromolecules 2004, 5, $1392-1398$

(26) Basle, E.; Joubert, N.; Pucheault, M. Protein Chemical Modification on Endogenous Amino Acids. Chem. Biol. 2010, 17, 213-227.

(27) Ai, H. W.; Henderson, J. N.; Remington, S. J.; Campbell, R. E. Directed Evolution of a Monomeric, Bright and Photostable Version of Clavularia cyan Fluorescent Protein: Structural Characterization and Applications in Fluorescence Imaging. Biochem. J. 2006, 400, 531-540.

(28) Lian, X.; Chen, Y.-P.; Liu, T.-F.; Zhou, H.-C. Coupling Two Enzymes into a Tandem Nanoreactor Utilizing a Hierarchically Structured MOF. Chem. Sci. 2016, 7, 6969-6973.

(29) Sassolas, A.; Blum, L. J.; Leca-Bouvier, B. D. Immobilization Strategies to Develop Enzymatic Biosensors. Biotechnol. Adv. 2012, 30, $489-511$.

(30) Homaei, A. A.; Sariri, R.; Vianello, F.; Stevanato, R. Enzyme Immobilization: An Update. J. Chem. Biol. 2013, 6, 185-205.

(31) Liu, A.; Yang, L.; Traulsen, C. H. -H.; Cornelissen, J. J. L. M. Immobilization of Catalytic Virus-like Particles in a Flow Reactor. Chem. Commun. 2017, 53, 7632-7634.

(32) Heller, A.; Feldman, B. Electrochemical Glucose Sensors and Their Applications in Diabetes Management. Chem. Rev. 2008, 108, 2482-2505.

(33) Shlyahovsky, B.; Li, D.; Katz, E.; Willner, I. Proteins Modified with DNAzymes or Aptamers Act as Biosensors or Biosensor Labels. Biosens. Bioelectron. 2007, 22, 2570-2576.

(34) Nicol, M. J.; Duke, F. R. Substrate Inhibition with Glucose Oxidase. J. Biol. Chem. 1966, 241, 4292-4293.

(35) Salvachúa, D.; Prieto, A.; Martínez, A. T.; Martínez, M. J. Characterization of a Novel Dye-decolorizing Peroxidase (DyP)-Type Enzyme from Irpex lacteus and Its Application in Enzymatic Hydrolysis of Wheat Straw. Appl. Environ. Microbiol. 2013, 79, 4316-4324. 
(36) Marabini, R.; Masegosa, I. M.; San Martin, M. C.; Marco, S.; Fernandez, J. J.; de la Fraga, L. G.; Vaquerizo, C.; Carazo, J. M. Xmipp: An Image Processing Package for Electron Microscopy. J. Struct. Biol. 1996, 116, 237-240.

(37) Scheres, S. H. RELION: Implementation of a Bayesian Approach to Cryo-EM Structure Determination. J. Struct. Biol. 2012, $180,519-530$.

(38) de la Rosa-Trevin, J. M.; Quintana, A.; Del Cano, L.; Zaldivar, A.; Foche, I.; Gutierrez, J.; Gomez-Blanco, J.; Burguet-Castell, J.; Cuenca-Alba, J.; Abrishami, V.; et al. Scipion: A Software Framework Toward Integration, Reproducibility and Validation in 3D Electron Microscopy. J. Struct. Biol. 2016, 195, 93-99.

(39) Pettersen, E. F.; Goddard, T. D.; Huang, C. C.; Couch, G. S.; Greenblatt, D. M.; Meng, E. C.; Ferrin, T. E. UCSF Chimera-A Visualization System for Exploratory Research and Analysis. J. Comput. Chem. 2004, 25, 1605-1612.

(40) Rohou, A.; Grigorieff, N. CTFFIND4: Fast and Accurate Defocus Estimation from Electron Micrographs. J. Struct. Biol. 2015, 192, 216-221.

(41) Shapiro, A. L.; Vinuela, E.; Maizel, J. V. Molecular Weight Estimation of Polypeptide Chains by Electrophoresis in SDSPolyacrylamide Gels. Biochem. Biophys. Res. Commun. 1967, 28, 815-820. 